\title{
COIL END DESIGN FOR SUPERCONDUCTING MAGNETS APPLYING DIFFERENTIAL GEOMETRY METHODS
}

\author{
B. Auchmann, S. Russenschuck
}

\begin{abstract}
An integrated design approach is used at CERN for the design and optimization of superconducting accelerator magnets, with the ROXIE program package as the key tool. The layout of the coil ends has proven in most cases to be the limiting factor for the magnets' quench performance. The objectives for coil end design are therefore to minimize the mechanical stress on the cables, to optimize the integrated multipole content and to limit the peak field enhancement. This paper introduces a new approach based on differential geometry methods that allows for the geometrical and mechanical optimization of $\cos n \theta \operatorname{coil}$ ends.
\end{abstract}




\title{
Coil End Design for Superconducting Magnets Applying Differential Geometry Methods
}

\author{
Bernhard Auchmann, Stephan Russenschuck \\ CERN-AT-MEL, 1211 Geneva 23, \\ E-mail: bernhard.auchmann/stephan.russenschuck@cern.ch
}

\begin{abstract}
An integrated design approach is used at CERN for the design and optimization of superconducting accelerator magnets, with the ROXIE program package as the key tool. The layout of the coil ends has proven in most cases to be the limiting factor for the magnets' quench performance. The objectives for coil end design are therefore to minimize the mechanical stress on the cables, to optimize the integrated multipole content and to limit the peak field enhancement. This paper introduces a new approach based on differential geometry methods that allows for the geometrical and mechanical optimization of $\cos n \theta$ coil ends.
\end{abstract}

\section{INTRODUCTION}

The coil shapes of the superconducting (SC) bending, focusing and corrector magnets for the Large Hadron Collider (LHC) at CERN were designed to approximate a $\cos n \theta$ current distribution with blocks of up to 26 rectangular or keystoned conductors. For the magnet design, an integrated approach has been adopted, with the ROXIE program package as the key tool. It combines a feature-based parametric mesh generator, the coupling method between finite elements and boundary elements (BEM-FEM), [3], and a variety of optimization algorithms and CAD/CAM interfaces. After the optimization of the coil cross-section and the shape optimization of the ferromagnetic yoke, the 3-dimensional geometry of the coil ends needs to be determined. Local peak field enhancements combined with a weaker mechanical support structure result in so-called training quenches in the coil ends, thus, necessitating an optimum layout of the support structure. The design objectives are as follows:

1) Minimization of mechanical stress on the cables.

2) Mimimization of the multipole content of the integrated field.

3) Limitation of the peak field enhancement.

4) Generation of CAD data for the 5-axis CNC machining of glass-epoxy filling pieces (end-spacers) which are wound into the coild ends.

Constructive solid geometry (CSG) program packages can go a long way in an exlusively geometry-driven coil end design. The interdependence of geometric design and magnetic field quality, however, makes the integration of the geometrical design process into an overall framework of magnet design and optimization, as provided by ROXIE, advisable.

The objectives 2 and 3 are met by optimizing the axial position of the individual cable blocks. This paper will present a differential-geometry based approach to the objectives 1 and 4,
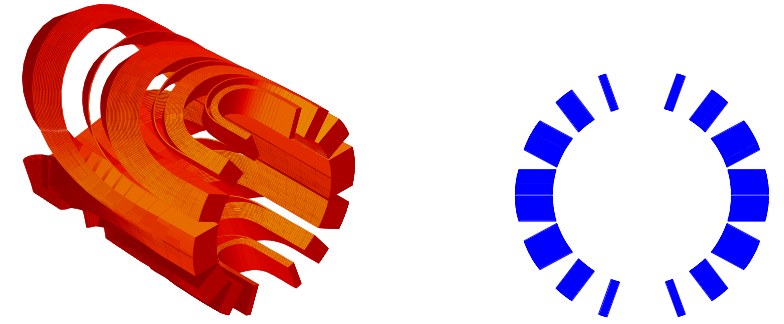

Fig. 1. Left: Coil end of the Main Corrector Bending (MCB) magnet, connection side. Right: Cross-section of the MCB dipole. Note the cable distribution that is designed to approximate a $\cos n \theta$ current distribution in a shell around the magnet aperture.

i.e., the geometrical and mechanical optimization of coil ends and spacers. Objective 1 can be reformulated in terms of the minimum strain energy $E$ :

$$
E=\frac{1}{2} \int_{0}^{s_{\mathrm{c}}}\left(f_{\tau} \tau(s)^{2}+f_{\kappa_{\mathrm{n}}} \kappa_{\mathrm{n}}(s)^{2}+f_{\kappa_{\mathrm{g}}} \kappa_{\mathrm{g}}(s)^{2}\right) \mathrm{d} s,
$$

wherein $s_{\mathrm{c}}$ is the cable length. The curvature parameters $\tau, \kappa_{\mathrm{n}}$, $\kappa_{\mathrm{g}}$ denote the torsion, the normal curvature and the geodesic curvature. These parameters are the local form of the global parameters which are called torsion, easy way bend (bending over the broad side of a rectangular shaped cable) and hard way bend (bending over the narrow side of a cable). The flexural rigidities of the cable, $f_{\tau}, f_{\kappa_{\mathrm{n}}}, f_{\kappa_{\mathrm{g}}}$, are material and cable-type related constants to be measured. Whereas the Rutherford type cables in the LHC main magnets allow some bending over their narrow side, the conductors of the corrector magnets are made of ribbons of up to 26 quadratic wires which allow hardly any geodesic curvature at all. In this case

$$
f_{\kappa_{\mathrm{g}}} \gg f_{\tau}, f_{\kappa_{\mathrm{n}}}
$$

so that the objective of minimum strain energy in a cable can be simplified to requiring that $\int_{0}^{s_{\mathrm{c}}} \kappa_{\mathrm{g}}^{2} \mathrm{~d} s$ be minimized (while keeping peak values of $\tau, \kappa_{\mathrm{n}}$ and $\kappa_{\mathrm{g}}$ below some critical values).

In Section II a mathematical model of the ribbons is presented, which allows to calculate and adjust the curvature parameters, in Section III information on the software implementation is given and in Section IV the experience gained in the design of the MCB dipole corrector magnet is presented. Finally, the combination of the coil end design algorithms with numerical field calculation is demonstrated. 


\section{Mathematical Model}

At first approximation, the theory of strpis describes a ribbontype cable that is bent into the three-dimensional space as a developable surface, i.e., it can be (un)bent into a plane. (These surfaces can essentially be made of a strip of paper if we assume sufficient smoothness and exclude possible ways of crumpling.) Bending is the act of continuous deformation of a surface under the constraint that arclengths of arbitrary curves in the surface be preserved. The baseline $\mathbf{r}: \mathbb{R} \rightarrow E^{3}$, $s \mapsto \mathbf{r}(s)$ constitutes one edge of this bent strip in Euclidian 3 -space. A developable surface is parameterized by

$$
\mathbf{p}(s, \lambda)=\mathbf{r}(s)+\lambda \mathbf{g}(s),
$$

where $\mathrm{g}(s)$ is the field of generator vectors (or rulings) and where $s$ and $t$ are parameters. Without loss of generality we select the arc-length on the baseline as the parameter $s$. The generators are straight lines (set $s=$ const.) which are found to be the intersections of successive tangent planes $F(s)$ and $F(s+\mathrm{d} s)$ to the strip surface, [5]. A tangent plane is uniquely defined by:

$$
F(s)=\left(\mathbf{r}_{0}-\mathbf{r}(s)\right) \cdot \mathbf{n}(s)=0,
$$

where $\mathbf{n}(s)$ is a normal vector to the strip in $\mathbf{r}(s)$ and $\mathbf{r}_{0}$ is some point in the tangent plane. Consequently it is required that $F(s)=F(s+\Delta s)=0$, and hence

$$
F(s)=\frac{F(s+\Delta s)-F(s)}{\Delta s}=0 .
$$

With $\Delta s \rightarrow 0$ we get

$$
F(s)=0, \quad \frac{\mathrm{d} F(s)}{\mathrm{d} s}=0 .
$$

We ought to determine points $\mathbf{r}_{0}$ in $E^{3}$ that fulfill the above equations. To find a solution to this problem, a field of trihedrals is assigned to the baseline, which is sometimes denoted a 'moving' trihedral. A trihedral is an orthonormal set of vectors $\{\mathbf{t}(s), \mathbf{n}(s), \mathbf{b}(s)\}$, where $\mathbf{t}(s)=\frac{\mathrm{d} \mathbf{r}(s)}{\mathrm{d} s}=\mathbf{r}^{\prime}(s)$ is the tangent vector, $\mathbf{n}(s)$ is the normal vector, and $\mathbf{b}(s)=$ $\mathbf{n}(s) \times \mathbf{t}(s)$ is called the binormal vector. The vectors $\mathbf{t}(s)$ and $\mathbf{b}(s)$ span the tangent plane to the strip surface.

In the following, the $s$-dependency of curvature parameters and vectors will be omitted in the notation. The above introduced curvature parameters read (c.f. Appendix)

$$
\begin{aligned}
\tau & =\mathbf{b} \cdot \mathbf{n}^{\prime}=\vartheta_{\mathbf{t}}^{\prime}, \\
\kappa_{\mathrm{g}} & =\mathbf{t} \cdot \mathbf{b}^{\prime}=\vartheta_{\mathbf{n}}^{\prime}, \\
\kappa_{\mathrm{n}} & =\mathbf{n} \cdot \mathbf{t}^{\prime}=\vartheta_{\mathbf{b}}^{\prime} .
\end{aligned}
$$

Given a baseline and a field of trihedrals, the three curvature parameters denote the differential twist angles $\mathrm{d} \vartheta_{\mathbf{t}, \mathbf{b}, \mathbf{n}}$ around the three trihedral axes as the trihedral moves along the baseline by d $s$, compare Fig. 2 .

Now we use the generalized Frenet-Serret Equations for strips, which are also derived in the Appendix:

$$
\begin{aligned}
\mathbf{t}^{\prime} & =\kappa_{\mathrm{n}} \mathbf{n}-\kappa_{\mathrm{g}} \mathbf{b}, \\
\mathbf{n}^{\prime} & =\tau \mathbf{b}-\kappa_{\mathrm{n}} \mathbf{t}, \\
\mathbf{b}^{\prime} & =\kappa_{\mathrm{g}} \mathbf{t}-\tau \mathbf{n} .
\end{aligned}
$$

For $\kappa_{\mathrm{g}}=0$ Eqs. $(8 \mathrm{a}-8 \mathrm{c})$ yield the well-known Frenet-Serret Equations of curves. Deriving Eq. (4) yields

$$
F^{\prime}(s)=\left(\mathbf{r}_{0}-\mathbf{r}\right) \cdot\left(\tau \mathbf{b}-\kappa_{\mathrm{n}} \mathbf{t}\right)=0 .
$$

Comparing this condition for points on the generator lines with Eq. (3), a valid solution for the generator vectors is given by:

$$
\mathbf{g}=\tau \mathbf{t}+\kappa_{\mathrm{n}} \mathbf{b}
$$

Given a baseline (e.g. an ellipse on a cylinder) and a field
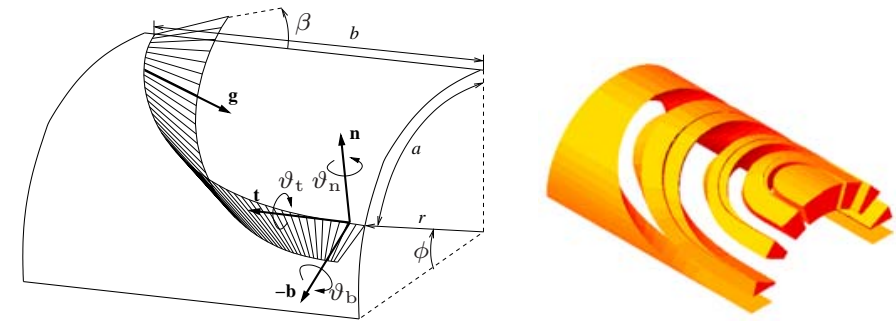

Fig. 2. Left: A strip bent along a baseline drawn on a cylinder of radius $r$, together with the generator lines of the developable surface. Note the right-hand rule orientation of the trihedral $(-\mathbf{b})$. Right: New design for the connection side endspacers of the MCB SC dipole magnet.

of trihedrals on that baseline, the curvature parameters can be derived according to Eqs. $7 \mathrm{a}-7 \mathrm{c}$ and the parameterization of a strip surface can be done according to Eq. 3 and 10. The task now consists of finding a field of trihedrals such that the peak values of curvature parameters are a minimum and that the integral value of the squared geodesic curvature is as small as possible. The Frenet Frame, [1],

$$
\mathbf{t}=\mathbf{r}^{\prime}, \quad \mathbf{n}=-\frac{1}{\kappa_{\mathrm{n}}} \mathbf{t}^{\prime}, \quad \mathbf{b}=\mathbf{n} \times \mathbf{t},
$$

guarantees zero geodesic curvature over the entire baseline; the strip then lies in the envelope of the rectifying planes $(\operatorname{span}\{\mathbf{t}, \mathbf{b}\})$ and the space curve becomes a geodesic (shortest path between two points on the surface).

For practical coil winding of superconducting magnets, however, the Frenet Frame is not ideal because of two reasons:

1) The coils are wound on a cylinder-shaped winding mandrel and the coil ends must match the magnet's straight section (determined by the 2-dimensional field quality optimization in the straight section). The Frenet Frame, however, requires the cable to meet the straight section in a radial position on the mandrel.

2) Bending a plane strip into a defined position consists in bending the strip over its successive generator lines, compare Fig. 3. The absolute value of the generator vector, $\sqrt{\tau^{2}+\kappa_{\mathrm{n}}^{2}}$, denotes the differential angle by which to bend the strip. It follows that the generator lines must not intersect in the surface of the cable in order to avoid tearing it. The locus of intersection of successive generators is called the edge of regression. The geodesic strip generally does not account for the absence of such intersections of generators in the cable surface (henceforth denoted an edge-of-regression violation). 
3) Even if the innermost cable of a block was wound as a geodesic strip with zero geodesic curvature, the remaining block that needs to be wound onto the first cable, would generally not find itself in an optimum position. This effect is further discussed in Section IV.

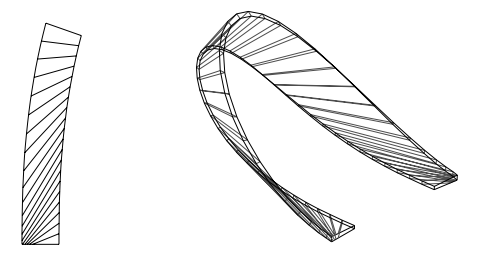

Fig. 3. Left: Half of the cable developed into a plane. Note the geodesic curvature in the developed strip, the generator lines, and in particular the first 6 intersecting generators. Right: Cable bent over the generator lines.

The application of an additional twist $\vartheta_{\mathbf{t}}^{*}(s)$ around the tangent vector $\mathbf{t}$ can be a solution to both of the above problems. It results, however, in the generation of geodesic curvature. From Eq. (7 a) it follows directly that

$$
\tau^{*}=\tau+\vartheta_{\mathbf{t}}^{* \prime}
$$

Inserting

$$
\begin{aligned}
\mathbf{t}^{*} & =\mathbf{t}, \\
\mathbf{n}^{*} & =\cos \vartheta_{\mathbf{t}}^{*} \mathbf{n}+\sin \vartheta_{\mathbf{t}}^{*} \mathbf{b}, \\
\mathbf{b}^{*} & =-\sin \vartheta_{\mathbf{t}}^{*} \mathbf{n}+\cos \vartheta_{\mathbf{t}}^{*} \mathbf{b},
\end{aligned}
$$

in Eqs. (7b) and (7c) yields

$$
\begin{aligned}
& \kappa_{\mathrm{g}}^{*}=\cos \vartheta_{\mathbf{t}}^{*} \kappa_{\mathrm{g}}+\sin \vartheta_{\mathbf{t}}^{*} \kappa_{\mathrm{n}}, \\
& \kappa_{\mathrm{n}}^{*}=-\sin \vartheta_{\mathbf{t}}^{*} \kappa_{\mathrm{g}}+\cos \vartheta_{\mathbf{t}}^{*} \kappa_{\mathrm{n}} .
\end{aligned}
$$

With Eqs. (11), (12) and (14 a-14 b), the mathematical model now accounts for a start configuration on a given baseline and yields the necessary theory for the design optimization.

\section{IMPLEMENTATION}

In practice the cables are wound on a cylindrical winding mandrel. The baseline on the mandrel is chosen to be a pseudo hyper-ellipse $\frac{x^{n}}{a^{n}}+\frac{y^{2}}{b^{2}}=1$ of order $n$ and ellipticity $f=\frac{b}{a}$, compare Fig. 2. With these design parameters, the given radius of the winding mandrel and the fixed angular position at the onset of the bend, the new procedure follows the subsequent steps:

1) The Frenet Frame is calculated analytically.

2) Additional twist is applied to match the magnets cross section at the onset of the bend.

3) The entire cable block geometry is generated, including optional inter-turn insulation or wedges.

4) The curvature parameters are calculated at every discretization point and in every cable of the coil block.

The following objectives for the design optimization can be calculated:

1) Integrated squared geodesic curvature over each coil block.
2) Maximum curvature parameter in each coil block.

3) A parameter indicating an edge-of-regression violation within the strip surface.

An objective weighting function is set up and solved using a deterministic optimization algorithm. The following design variables are considered:

1) Ellipticity of the baseline.

2) Order of the hyper-ellipse on the cylinder (shape of the baseline).

3) Angle $\beta$ between the innermost cable turn and the mandrel at the apex of the baseline.

4) Four knots of a cubic spline function allowing for the local adjustment of the cable torsion between the onset of the coil end and the baseline apex according to Eqs. (12-14b).

To detect an edge-of-regression within the cable width, the length of the generators from the baseline to the outer edge of the cable must be determined. In the 3-dimensional model this is a non-trivial task. The inner theory of surfaces suggests to determine the geodesic that, form the point $\mathbf{r}$ strives towards the outer edge with $\mathbf{b}$ tangent to the geodesic. Given a parameter representation $\mathbf{p}(s, \lambda)$ of the surface, the geodesic is found by the means of variation calculus. This computationally challenging task can be avoided by making use of two bending invariances:

1) The angle between $t$ and $g$.

2) The geodesic curvature $\kappa_{g}$.

In the unbent (plane) strip, where $\rho_{\mathrm{g}}=\frac{1}{\kappa_{\mathrm{g}}}$ is the radius of curvature of the inner edge, the generator lengths can be determined by fundamental geometric operations, compare Fig. 3 (left). A continuous parameter to indicate the severity of edge-of-regression violations is given by the integrated magnitude of the generator vectors over the baseline where generator intersections within the cable surface occur. Unlike the outer cable edge, the generators' lengths to the outer endspacer edge are easy to compute. In the 3-dimensional model they are determined by the intersection of the generators with a cylinder of the outer radius of the end-spacer.

The obtained algorithm is compatible with the routines previously implemented in ROXIE. For instance the design of connection side coil ends benefits from the existing routines. The simultaneous optimization of the left and right parts of cross-over turns, coupled only by the angle $\beta$ has lead to satisfactory results for both sides of the asymmetric end. The post-processing and the existing $\mathrm{CAD} / \mathrm{CAM}$ interfaces can also be used.

\section{RESULTS}

The mathematical model and the design procedure has been validated at the coil end design of the SC Main Corrector Bending (MCB) magnet for the LHC. This coil end design was particularly difficult due to a large number of conductors per coil block (maximum 26) and due to the brittle nature of the ribbon conductor that does not support geodesic curvature. Previous designs based only on global parameters such as 
the constant arc length of inner and outer cable edge had led to an unacceptable lift-off of the turns from the winding mandrel. A different approach accounted only for a minimum

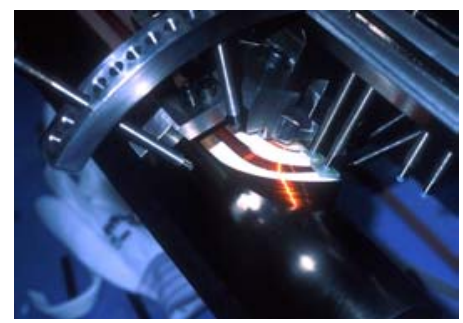

Fig. 4. Winding process of the MCB dipole corrector coil ends. Note the tooling for the clamping of the outer turns, especially needed in the position where Fig. 5 (up) shows a maximum in geodesic curvature.

geodesic curvature in the innermost cable of each block. With the new algorithm it could be shown that in this case the strain energy of the outermost turn in a block of 26 cables is 18.4 times higher than in the first turn. This, again, lead to an unacceptable lift-off of the outer turns from the winding mandrel. The effect can be understood as the cables' attempt to 'ease' the strain energy imposed on them by leaving the foreseen baseline. The cable blocks needed to be clamped onto the mandrel, thus, forcing the cable into a position of higher geodesic curvature and strain energy, compare Figs. 4 and 5.

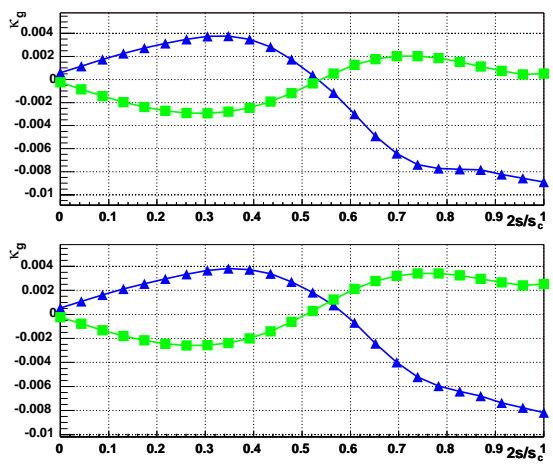

Fig. 5. Geodesic curvature in the innermost cable (squares) and the outermost cable (triangles) in the second block of the MCB SC corrector magnet, containing 26 conductors. Up: Result of a minimization of the geodesic curvature in the innermost cable only. Down: Optimum solution to the minimization of the total block strain energy.

The minimization of the total strain energy in each coil block, balances the inevitable local geodesic curvature between the inner and outer cables. As compared to the case where only the geodesic curvature of the innermost turn was minimized, the total block strain energy could be reduced by a factor of 5. Fig. 5 illustrates the distribution of geodesic curvature in the innermost and outermost turns for both cases. A threedimensional BEM-FEM calculation was performed in order to calculate the variation of the multipole field errors in the end region of the two-in-one magnet assembly. The shape of the end spacers, see Fig. 2, was then transferred into a CAD system in order to produce spacers for winding trials. The new coil end design will now be adopted for the series production of the magnets.
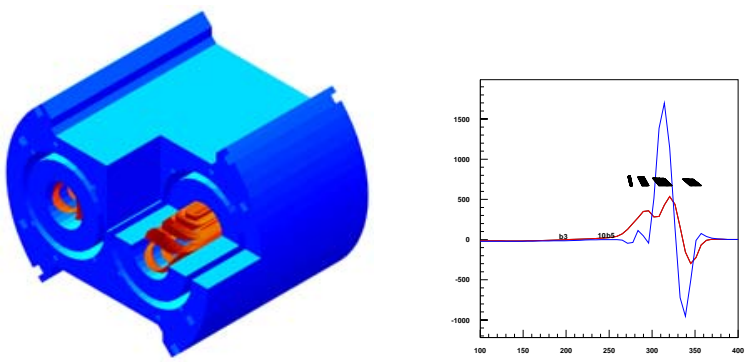

Fig. 6. Left: 3d-model with iron yoke and iron support structure of the MCB dipole corrector magnet. The iron structure has been cut for better visibility. Right: Multipole errors in the coil ends ( at $17 \mathrm{~mm}$ reference radius in units $10^{-} 4$ related to the main field) as a function of the $z$ position. $z=0$ is the center of the magnet.

\section{APPENDIX}

The derivation of the curvature parameters and the generalized Frenet-Serret equations follows [1].

Let $\left\{\mathbf{a}_{1}(s), \mathbf{a}_{2}(s), \mathbf{a}_{3}(s)\right\}$ be a one-parameter family of orthonormal sets of vectors, i. e.,

$$
\mathbf{a}_{j} \cdot \mathbf{a}_{k}=\delta_{j k},
$$

where, again, we omit the parameter in the notation. We rewrite the derivatives over $s$ in terms of linear combinations of the trihedral vectors:

$$
\mathbf{a}_{j}^{\prime}=\sum_{k=1}^{3} \mathbf{a}_{k} \omega_{j k},
$$

where the coefficients read

$$
\omega_{j k}=\mathbf{a}_{j}^{\prime} \cdot \mathbf{a}_{k} .
$$

Differentiation of Eq. (15) yields

$$
\mathbf{a}_{j}^{\prime} \cdot \mathbf{a}_{j}=0 \quad \text { and } \quad \mathbf{a}_{j}^{\prime} \cdot \mathbf{a}_{k}+\mathbf{a}_{k}^{\prime} \cdot \mathbf{a}_{j}=0,
$$

which implies that

$$
\omega_{j j}=0 \quad \text { and } \quad \omega_{j k}=-\omega_{k j} .
$$

Assuming that $\mathbf{a}_{1}=\mathbf{r}^{\prime}$ and $\mathbf{a}_{2}=\mathbf{n}, \mathbf{a}_{3}=\mathbf{b}$ we may set $\omega_{23}=\tau, \omega_{31}=\kappa_{\mathrm{g}}$ and $\omega_{12}=\kappa_{\mathrm{n}}$. Eq. (16) then yields the generalized Frenet-Serret equations $(8 a-8 c)$, whereas Eq. (17) implies Eqs. (7c - 7c).

\section{REFERENCES}

[1] W. Blaschke, Einführung in die Differentialgeometrie, 2. Auflg., SpringerVerlag, Berlin 1960

[2] J. M. Cook, "Strain Energy Minimization in SSC Magnet Winding", IEEE Transactions on Magnetics, Vol. 27, No. 2, March 1991

[3] S. Kurz and S. Russenschuck, "The Application of the BEM-FEM Coupling Method for the Accurate Calculation of Fields in Superconducting Magnets", Electrical Engineering, 1999

[4] S. Russenschuck and T. Tortschanoff, "Mathematical Optimization of Superconducting Accelerator Magnets", IEEE Transactions on Magnetics, Vol-Mag 30, No. 5, 1994, LHC-Note 246, CERN Geneva

[5] C. E. Weatherburn, Differential Geometry of Three Dimensions, University Press, Cambridge 1961 